\title{
Charmed and strange baryon resonances with heavy-quark spin symmetry *
}

\author{
O. Romanets ${ }^{\dagger a}$, L. Tolos ${ }^{b, a}$, C. García-Recio ${ }^{c}$, J. Nieves $^{d}$, L. L. Salcedo ${ }^{c}$, \\ R. G. E. Timmermans ${ }^{a}$ \\ ${ }^{a}$ KVI, University of Groningen \\ Zernikelaan 25, 9747AA Groningen, The Netherlands \\ ${ }^{b}$ Institut de Ciències de l'Espai (IEEC/CSIC) \\ Campus Universitat Autònoma de Barcelona, Facultat de Ciències, \\ Torre C5, E-08193 Bellaterra, Spain \\ ${ }^{c}$ Departamento de Física Atómica, Molecular y Nuclear, and Instituto Carlos I de Física Teórica \\ y Computacional, Universidad de Granada \\ E-18071 Granada, Spain \\ ${ }^{d}$ Instituto de Física Corpuscular (centro mixto CSIC-UV), Institutos de Investigación de Paterna \\ Aptdo. 22085, 46071, Valencia, Spain \\ E-mail: 0. romanets@rug.nI
}

\begin{abstract}
Charmed and strange baryon resonances that are generated dynamically are studied within a unitary baryon-meson coupled-channel model, which incorporates heavy-quark spin symmetry. The extension of the SU(3) Weinberg-Tomozawa chiral Lagrangian to SU(8) spin-flavor symmetry plus a suitable symmetry breaking is used. The model produces resonances with negative parity from $s$-wave interaction of pseudoscalar and vector mesons with $1 / 2^{+}$and $3 / 2^{+}$baryons. Some of our dynamically generated states can be readily assigned to resonances found experimentally, while others do not have a straightforward identification and require the compilation of more data and also refinements of the model.
\end{abstract}

Sixth International Conference on Quarks and Nuclear Physics

April 16-20, 2012

Ecole Polytechnique, Palaiseau, Paris

${ }^{*}$ This research was supported by DGI and FEDER funds, under contracts FIS2011-28853-C02-02, FIS2011-24149, FPA2010-16963 and the Spanish Consolider-Ingenio 2010 Programme CPAN (CSD2007-00042), by Junta de Andalucía grant FQM-225, by Generalitat Valenciana under contract PROMETEO/2009/0090 and by the EU HadronPhysics2 project, grant agreement n. 227431. O.R. and L.T. wishes to acknowledge support from the Rosalind Franklin Fellowship. L.T. acknowledges support from Ramon y Cajal Research Programme, and from FP7-PEOPLE-2011-CIG under contract PCIG09-GA-2011-291679.

† Speaker. 


\section{Introduction}

The observation of new charmed and strange baryon resonances and the plausible explanation of their nature is an active topic of research. Data about such states comes from CLEO, Belle, BaBar and other experiments, such as the planned PANDA and CBM at the FAIR facility at GSI. Those future experiments, which involve studies of charm physics, open the possibility for more data in the near future. The ultimate goal is to understand whether those new states can be described with the usual three-quark baryon or quark-antiquark meson interpretation or, alternatively, qualify better as hadron molecules.

Recent approaches based on coupled-channel dynamics have proven to be quite successful in describing the existing experimental data. In particular, unitarized coupled-channel methods have been applied in the baryon-meson sector with the charm degree of freedom [1, 2, 3, 4], partially motivated by the analogy between the $\Lambda(1405)$ and the $\Lambda_{c}(2595)$. Other existing coupled-channel approaches are based on the Jülich meson-exchange model [5] or on the hidden gauge formalism [6].

However, those previous models are not consistent with heavy-quark spin symmetry [7], which is a proper QCD symmetry that appears when the quark masses, such as the charm mass, become larger than the typical confinement scale. Aiming to incorporate heavy-quark symmetry, an SU(8) spin-flavor symmetric model has recently been developed [8, 9], which includes vector mesons similarly to the SU(6) approach developed in the light sector of Ref. [10].

The objective of this work is to study dynamically generated baryon resonances using heavyquark spin symmetry constraints. We focus on charm $C=1$ and strangeness $S=-2,-1$ and 0 , as well as on sectors with $C=2$ and 3. We therefore use the model of Ref. [8] and, as novelty, we pay special attention to the pattern of spin-flavor symmetry breaking. Flavor SU(4) is not a good symmetry in the limit of a heavy charm quark. Therefore, instead of the breaking pattern $\mathrm{SU}(8) \supset \mathrm{SU}(4)$, in this work we consider the pattern $\mathrm{SU}(8) \supset \mathrm{SU}(6)$, since the light spin-flavor group $\mathrm{SU}(6)$ is decoupled from heavy-quark transformations. This allows us to implement heavyquark spin symmetry in the analysis and to unambiguously identify the corresponding multiplets among the resonances generated dynamically [11]. At the same time, we are also able to assign approximate heavy $\mathrm{SU}(8)$ and light $\mathrm{SU}(6)$ spin-flavor multiplet labels to the states.

\section{Framework}

For the baryon-meson interaction we use the model of Refs. [8, 9]. This model is based on an extension of the Weinberg-Tomozawa (WT) SU(3) chiral Lagrangian. The channel space includes charmed vector mesons and $3 / 2^{+}$baryons, in addition to pseudoscalar mesons and $1 / 2^{+}$baryons. The model obeys spin-flavor symmetry and also heavy-quark spin symmetry (HQSS) in the sectors studied in this work. Schematically,

$$
\mathscr{L}_{\mathrm{WT}}^{S U(8)}=\frac{1}{f^{2}}\left[\left[M^{\dagger} \otimes M\right]_{\mathbf{6 3}_{\mathbf{a}}} \otimes\left[B^{\dagger} \otimes B\right]_{\mathbf{6 3}}\right]_{\mathbf{1}},
$$

where $M$ is the $\mathbf{6 3}$ meson multiplet and $B$ is the $\mathbf{1 2 0}$ baryon multiplet of $\mathrm{SU}(8)$.

In the $s$-channel, the baryon-meson space reduces into four SU(8) irreps, from which two multiplets 120 and $\mathbf{1 6 8}$ are the most attractive ones, while the 4752-plet is weakly attractive and 
the 2520-plet is repulsive. As a consequence, dynamically-generated baryon resonances are most likely to occur within the $\mathbf{1 2 0}$ and $\mathbf{1 6 8}$ sectors. Therefore, only states that belong to these two representations are studied.

To take into account the breaking of flavor symmetry introduced by the heavy charmed quark, we consider the reduction $\mathrm{SU}(8) \supset \mathrm{SU}(6) \times \mathrm{SU}_{C}(2) \times \mathrm{U}_{C}(1)$, where $\mathrm{SU}(6)$ is the spin-flavor group for three flavors and $\mathrm{SU}_{C}(2)$ is the rotation group of quarks with charm. We consider only $s$-wave interactions so $J_{C}$ is just the spin carried by the charmed quarks or antiquarks. Finally, $\mathrm{U}_{C}(1)$ is the group generated by the charm quantum number $C$. Further, the $\mathrm{SU}(6)$ multiplets can be reduced under $\mathrm{SU}(3) \times \mathrm{SU}_{l}(2)$, the factor $\mathrm{SU}_{l}(2)$ referring to the spin of the light quarks. In order to connect with the labeling $(C, S, I, J)$ based on isospin multiplets ( $S$ is the strangeness, $I$ the isospin, $J$ the spin), we further reduce $\mathrm{SU}_{l}(2) \times \mathrm{SU}_{C}(2) \supset \mathrm{SU}(2)$ where $\mathrm{SU}(2)$ refers to the total spin $J$, that is, we couple the spins of light and charmed quarks to form SU(3) multiplets with well-defined $J$.

The tree-level baryon-meson interaction of the SU(8)-extended WT interaction reads

$$
V_{i j}(s)=D_{i j} \frac{2 \sqrt{s}-M_{i}-M_{j}}{4 f_{i} f_{j}} \sqrt{\frac{E_{i}+M_{i}}{2 M_{i}}} \sqrt{\frac{E_{j}+M_{j}}{2 M_{j}}} .
$$

Here, $i$ and $j$ are the outgoing and incoming baryon-meson channels, respectively. $M_{i}, E_{i}$ and $f_{i}$ stand, respectively, for the mass, the center-of-mass energy of the baryon, and the meson decay constant in the $i$ channel. $D_{i j}$ are the matrix elements coming from the $\mathrm{SU}(8)$ group structure of the coupling for the various $C S I J$ sectors. In order to calculate the scattering amplitudes, $T_{i j}$, we solve the on-shell Bethe-Salpeter equation in coupled channels using the interaction matrix $V$ as kernel:

$$
T(s)=\frac{1}{1-V(s) G(s)} V(s) .
$$

$G(s)$ is a diagonal matrix containing the baryon-meson propagator for each channel. The quantities $D, T, V$, and $G$ are matrices in coupled-channel space. The loop function $G_{i i}^{0}(s)$ is logarithmically ultraviolet divergent and is regularized by means of the subtraction point regularization [12].

The dynamically-generated baryon resonances can be obtained as poles of the scattering amplitudes. The mass and the width of the resonance can be found from the position of the pole on the complex energy plane. Close to the pole, the $T$-matrix behaves as

$$
T_{i j}(s) \approx \frac{g_{i} g_{j}}{\sqrt{s}-\sqrt{s_{R}}},
$$

and in this way the coupling constants $g_{i}$ to different meson-baryon channels can be found.

The matrix elements $D_{i j}$ display exact $\mathrm{SU}(8)$ invariance, but this symmetry is severely broken in nature, so we implement a symmetry-breaking mechanism. The symmetry breaking pattern, with regards to flavor, follows the chain $\mathrm{SU}(8) \supset \mathrm{SU}(6) \supset \mathrm{SU}(3) \supset \mathrm{SU}(2)$, where the last group refers to isospin. The symmetry breaking is introduced by means of a deformation of the mass and decay constant parameters. This allows us to assign well-defined SU(8), SU(6), and SU(3) labels to the resonances and to find HQSS invariant states.

\section{Dynamically generated charmed and strange baryon states}

Our model obtains the dynamically generated states in different charm and strange sectors, namely $C=1,2,3$ and the corresponding strangeness numbers [11]. We have assigned to some of 
them a tentative identification with known states from the PDG [13]. This identification is made by comparing the data from the PDG on these states with the information we extract from the poles, namely the mass, width and, most important, the couplings. The couplings give us valuable information on the structure of the state and on the possible decay channels and their relative strength.

One of the sectors that we study is $C=1, S=0, I=0$, which corresponds to $\Lambda_{c}$ spin- $1 / 2$ and spin-3/2 states. Results for $C=1$ and $S=0$ were reported previously in Ref. [8]. However, the analysis of the dynamically generated states in terms of the attractive $\mathrm{SU}(8) \supset \mathrm{SU}(6) \supset \mathrm{SU}(3) \supset$ $\mathrm{SU}(2)$ multiplets was not done in this previous reference. Here we are able to assign $\mathrm{SU}(8), \mathrm{SU}(6)$, and SU(3) labels to the resonances. Simultaneously, we also classify the resonances into HQSS multiplets, in practice doublets and singlets. We obtain the three lowest-lying states of Ref. [8] in this sector. However, those states appear with slightly different masses due to the different subtraction point, and different $D_{s}$ and $D_{s}^{*}$ meson decay constants. The experimental $\Lambda_{c}(2595)$ resonance can be identified with the $\mathbf{2 1}_{2,1}$ pole ${ }^{1}$ that we found around $2618.8 \mathrm{MeV}$, as similarly done in Ref. [8]. The width in our case is, however, smaller than the experimental value, but we have not included the three-body decay channel $\Lambda_{c} \pi \pi$, which already represents almost one third of the decay events [13]. We also observe a second broad $\Lambda_{c}$ resonance at $2617 \mathrm{MeV}$ with a large coupling to the open channel $\Sigma_{c} \pi$, very close to $\Lambda_{c}(2595)$. This is precisely the same two-pole pattern found in the charmless $I=0, S=-1$ sector for the $\Lambda(1405)$ [14]. The third spin- $1 / 2 \Lambda_{c}$ resonance with a mass of $2828 \mathrm{MeV}$ is mainly originated by a strong attraction in the $\Xi_{c} K$ channel but it cannot be assigned to any experimentally known resonance. We also find one spin-3/2 resonance in this sector located at $(2666.6-i 26.7 \mathrm{MeV})$. Similarly to the Ref. [8] this resonance is assigned to the experimental $\Lambda_{c}(2625)$. A similar resonance was found at $2660 \mathrm{MeV}$ in the $t$-channel vector-exchange model of Ref. [3]. The novelty of our calculation is that we obtain a non-negligible contribution from the baryon-vector meson channels to the generation of this resonance, as already observed in Ref. [8].

The three $\Sigma_{c}$ resonances obtained for $C=1, S=0, I=1, J=1 / 2$ with masses $2571.5,2622.7$ and $2643.4 \mathrm{MeV}$ and widths $0.8,188.0$ and $87.0 \mathrm{MeV}$ respectively are predictions of our model, since no experimental data have been observed in this energy region. Our predictions here nicely agree with the three lowest lying resonances found in Ref. [8]. The model of Ref. [4] predicts the existence of two resonances with $C=1, S=0, I=1, J=\frac{1}{2}$. In this reference, the first one has a mass of $2551 \mathrm{MeV}$ with a width $0.15 \mathrm{MeV}$. It couples strongly to the $\Sigma D_{s}$ and $N D$ channels and, therefore, might be associated with the resonance $\Sigma_{c}(2572)$ with $\Gamma=0.8 \mathrm{MeV}$ of our model. Nevertheless, in our model this resonance couples most strongly to the other channels which incorporate vector mesons, such as $\Sigma^{*} D_{s}^{*}$ and $\Delta D^{*}$. The second resonance predicted in Ref. [4] has a mass of $2804 \mathrm{MeV}$ and a width of $5 \mathrm{MeV}$, and it cannot be compared to any of our results. We predict two spin-3/2 $\Sigma_{c}$ resonances. The first one, a bound state at $2568.4 \mathrm{MeV}$, lies below the threshold of any possible decay channel. This state is thought to be the charmed counterpart of the hyperonic $\Sigma(1670)$ resonance. While the $\Sigma(1670)$ strongly couples to $\Delta \bar{K}$ channel, this resonance is mainly generated by the analogous $\Delta D$ and $\Delta D^{*}$ channels. The second state at $2692.9-i 33.5$ $\mathrm{MeV}$ has no direct comparison with the available experimental data.

\footnotetext{
${ }^{1} R_{2 J_{C}+1, C}$ stands for $R$ irrep of SU(6).
} 
We also study the $C=1, S=-1, I=1 / 2$ sector for $\operatorname{spin} J=1 / 2$ and $J=3 / 2$. Those states are labeled by $\Xi_{c}$ and our model predicts the existence of nine states stemming from the strongly attractive 120 and $168 \mathrm{SU}(8)$ irreducible representations. Among them six states have spin $J=1 / 2$. In the energy range where these six states predicted by our model lie, three experimental resonances have been seen by the Belle, E687, and CLEO Collaborations: $\Xi_{c}(2645) J^{P}=3 / 2^{+}$ $[13,16], \Xi_{c}(2790) J^{P}=1 / 2^{-}[13,17]$ and $\Xi_{c}(2815) J^{P}=3 / 2^{-}[13,16]$. While $\Xi_{c}(2645)$ cannot be identified with any of our states due to the parity, the $\Xi_{c}(2790)$ might be assigned with one of the six resonances in the $J=1 / 2$ sector. The state $\Xi_{c}(2790)$ has a width of $\Gamma<12-15 \mathrm{MeV}$ and it decays to $\Xi_{c}^{\prime} \pi$, with $\Xi_{c}^{\prime} \rightarrow \Xi_{c} \gamma$. We assign it to the $2804.8-i 13.5 \mathrm{MeV}$ state found in our model because of the large $\Xi_{c}^{\prime} \pi$ coupling and the fact that a slight modification of the subtraction point can lower its position to $2790 \mathrm{MeV}$ and most probably reduce its width as it will get closer to the $\Xi_{c}^{\prime} \pi$ channel, the only channel open at those energies that couples to this resonance. Moreover, this seems to be a reasonable assumption in view of the fact that, in this manner, this $\Xi_{c}$ state is the HQSS partner of the $\Xi_{c}^{*}(2845)$ state, which we will identify with the $\Xi_{c}^{*}(2815)$ resonance of the PDG. It could be also possible to identify our pole at $2733 \mathrm{MeV}$ from the 168 irreducible representation with the experimental $\Xi_{c}(2790)$ state. In that case, one might expect that if the resonance position gets closer to the physical mass of $2790 \mathrm{MeV}$, its width will increase and it will easily reach values of the order of $10 \mathrm{MeV}$. As it was mentioned above, the only experimental $J^{P}=3 / 2^{-}$baryon resonance with a mass in the energy region of interest is $\Xi_{c}(2815)$. The full width is expected to be less than $3.5 \mathrm{MeV}$ for $\Xi_{c}^{+}$(2815) and less than $6.5 \mathrm{MeV}$ for $\Xi_{c}^{0}(2815)$, and the decay modes are $\Xi_{c+} \pi^{+} \pi^{-}, \Xi_{c 0} \pi^{+} \pi^{-}$. We obtain two resonances at $2819.7-i 16.2 \mathrm{MeV}$ and $2845.2-i 22.0 \mathrm{MeV}$, respectively, that couple strongly to $\Xi_{c}^{*} \pi$, with $\Xi_{c}^{*} \rightarrow \Xi_{c} \pi$. Allowing for this possible indirect three-body decay channel, we might identify one of them to the experimental result. This assignment is, indeed, possible for the state at $2845.2 \mathrm{MeV}$ if we slightly change the subtraction point. In this way, we will lower its position and reduce its width as it gets closer to the open $\Xi_{c}^{*} \pi$ channel.

According to our analysis, in the $C=1, S=-2, I=0, J=1 / 2$ sector there are three bound states $\left(\Omega_{c}\right)$ with masses $2810.9,2884.5$ and $2941.6 \mathrm{MeV}$. There is no experimental information on those excited states. However, our predictions are comparable to recent calculations of Refs. [4, 2]. In both these references, vector baryon-meson channels were not considered, breaking in this manner HQSS. In fact, it is worth noticing that the coupling to vector baryon-meson states plays an important role in the generation of the baryon resonances in this sector. Further, we obtain two spin-3/2 bound states $\Omega_{c}$ with masses 2814.3 and $2980.0 \mathrm{MeV}$, which mainly couple to $\Xi D^{*}$ and $\Xi^{*} D^{*}$, and to $\Xi_{c}^{*} \bar{K}$, respectively. As in the $J=1 / 2$ sector, no experimental information is available.

We also obtain baryon resonances with $C=2$ and 3 (see Ref. [11]). The appropriate numbers of strangeness of the states with charm 2 are $0\left(\Xi_{c c}\right)$ and $-1\left(\Omega_{c c}\right)$, which result from the group decomposition of the 120 and $168 \mathrm{SU}(8)$ representations. Finally, the $\Omega_{c c c}$ states with $C=3, S=$ $0, I=0$ are studied. At the moment no experimental information is available for those ones. To our knowledge, these are the first predictions in these sectors deduced from a model fulfilling HQSS.

\section{Summary}

In the present work, we have studied odd-parity charmed baryon resonances within a coupled- 
channel unitary approach that implements the characteristic features of HQSS. This is accomplished by extending the $\mathrm{SU}(3)$ WT chiral interaction to $\mathrm{SU}(8)$ spin-flavor symmetry and implementing a strong flavor symmetry breaking. We have discussed the predictions of the model for all $C=1$ strange sectors and have also looked at the $C=2$ and 3 predicted states. We have restricted our study to the 288 states (counting multiplicities in spin and isospin) that stem from the attractive 168 and 120 representations, for which we believe the predictions of the model are more robust. To identify these states, we have adiabatically followed the trajectories of the $\mathbf{1 6 8}$ and $\mathbf{1 2 0}$ poles, generated in a symmetric $\mathrm{SU}(8)$ world, when the symmetry is broken down to $\mathrm{SU}(6) \times \mathrm{SU}_{C}(2)$ and later $\mathrm{SU}(6)$ is broken down to $\mathrm{SU}(3) \times \mathrm{SU}(2)$. A first result of this work is that we have been able to identify the $\mathbf{1 6 8}$ and $\mathbf{1 2 0}$ resonances among the plethora of resonances predicted in Ref. [8] for the different strangeness $C=1$ sectors. Thus, we interpret the $\Lambda_{c}(2595)$ and $\Lambda_{c}(2625)$ as a members of the $\mathrm{SU}(8)$ 168-plet, and in both cases with a dynamics strongly influenced by the $N D^{*}$ channel, in sharp contrast with previous studies inconsistent with HQSS. There is scarce experimental information in all studied sectors. Other identifications correspond to the three-star $\Xi_{c}(2790)$ and $\Xi_{c}(2815)$ resonances in the $C=1, S=-1, I=1 / 2$ sectors. We believe that the rest of our predictions are robust, and will find experimental confirmation in the future. In particular, the program of PANDA of FAIR are of particular relevance.

\section{References}

[1] L. Tolos, J. Schaffner-Bielich and A. Mishra, Phys. Rev. C 70, 025203 (2004); M. F. M. Lutz and E. E. Kolomeitsev, Nucl. Phys. A 730, 110 (2004); T. Mizutani and A. Ramos, Phys. Rev. C 74, 065201 (2006).

[2] J. Hofmann and M. F. M. Lutz, Nucl. Phys. A 763, 90 (2005).

[3] J. Hofmann and M. F. M. Lutz, Nucl. Phys. A 776, 17 (2006).

[4] C. E. Jimenez-Tejero, A. Ramos and I. Vidana, Phys. Rev. C 80, 055206 (2009).

[5] J. Haidenbauer, G. Krein, U. G. Meissner and A. Sibirtsev, Eur. Phys. J. A 33, 107 (2007); J. Haidenbauer, G. Krein, U. G. Meissner and L. Tolos, Eur. Phys. J A 47, 18 (2011)

[6] J. -J. Wu, R. Molina, E. Oset and B. S. Zou, Phys. Rev. Lett. 105, 232001 (2010);

[7] N. Isgur, M. B. Wise, Phys. Lett. B232, 113 (1989); M. Neubert, Phys. Rept. 245, 259-396 (1994).

[8] C. Garcia-Recio et al., Phys. Rev. D 79, 054004 (2009).

[9] D. Gamermann et al., Phys. Rev. D 81, 094016 (2010).

[10] C. Garcia-Recio, J. Nieves and L. L. Salcedo, Phys. Rev. D 74, 034025 (2006); H. Toki, C. Garcia-Recio and J. Nieves, Phys. Rev. D 77, 034001 (2008).

[11] O. Romanets et al. ( Phys. Rev. D, in press), [hep-ph/1202.2239].

[12] J. Nieves and E. Ruiz Arriola, Phys. Rev. D 64, 116008 (2001).

[13] K. Nakamura et al. [Particle Data Group Collaboration], J. Phys. G G 37, 075021 (2010).

[14] D. Jido, J. A. Oller, E. Oset, A. Ramos and U. G. Meissner, Nucl. Phys. A 725, 181 (2003).

[15] C. Garcia-Recio, J. Nieves, E. Ruiz Arriola and M. J. Vicente Vacas, Phys. Rev. D 67, 076009 (2003).

[16] T. Lesiak et al. [ Belle Collaboration ], Phys. Lett. B665, 9-15 (2008).

[17] S. E. Csorna et al. [ CLEO Collaboration ], Phys. Rev. Lett. 86, 4243-4246 (2001). 